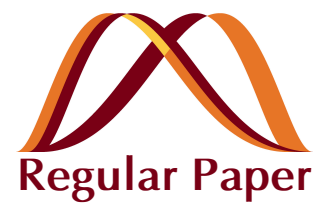

\title{
Reflectivity Improvement by Particle Neutralization in a Charged Particle-Type Electronic Display
}

\author{
Young-Cho Kim ${ }^{+}$ \\ Department of Electronic Engineering, Chungwoon University, Hongseong 350-701, Korea
}

Received October 24, 2012; Revised January 16, 2013; Accepted January 16, 2013

\begin{abstract}
Eight sample panels using an indium tin oxide(ITO)-coated glass substrate were fabricated, with barrier ribs formed of $55 \mu \mathrm{m}$ height and $10 \mu \mathrm{m}$ width. The upper and lower substrates were designed with the same panel condition, so a cell gap of $110 \mu \mathrm{m}$ was obtained. The charged particles in a cell consisted of $\mathrm{TiO}_{2}$ (for white color) or carbon black (black color), negative or positive charge control agents, and a polymer. The average diameter of the two types of particles was commonly $10 \mu \mathrm{m}$, and their $\mathrm{q} / \mathrm{m}$ value was $-4.5 \mu \mathrm{C} / \mathrm{g}$ and $+4.5 \mu \mathrm{C} / \mathrm{g}$, respectively. The electrically opposite particles mixed by an agitator were loaded into their cells by a simple particle-loading method. The discharging process proceeded at a humidity of $80 \%$ and a temperature of $30^{\circ} \mathrm{C}$. Reflectivity was measured depending on discharging time, and a hysteresis curve by bias voltage obtained for comparison between the neutralized and non-neutralized panel, in which the superior optical property of the neutralized panel was ascertained
\end{abstract}

Keywords: Reflective display, Neutralization, Discharging process, Hysteresis curve, Reflectivity, Optical property

\section{INTRODUCTION}

People have become accustomed to getting information from a paper-based book. Reading on paper is felt to be more convenient with less eyestrain, compared with reading on an emissive display screen. So, in recent years, many reflective electronic displays, which are called paper-like display or electronic paper (epaper), have been proposed, fabricated, and even commercialized in electronic books and signage, because of the advantages of low power consumption by the bi-stability phenomenon, low production cost by simple fabrication process, and ease of viewing by use of external light $[1,2]$.

From this point of view, a reflective electronic display using electrically positive and negative particles, namely quick response-liquid powder display (QR-LPD), is one of the most approachable reflective displays to encompass the above strong points $[3,4]$. In particular, the bi-stable property and fast response time is admirable. Despite these excellent advantages,

${ }^{\dagger}$ Author to whom all correspondence should be addressed: E-mail: yckim@chungwoon.ac.kr

Copyright $@ 2013$ KIEEME. All rights reserved. This is an open-access article distributed under the terms of the Creative Commons Atribution Non-Commercial
License (httr:///creativecommons.org distribution, and reproduction in any medium, provided the original work is properly cited. however, an unstable driving property can induce undesirable reliability, because of non-neutralized $\mathrm{q} / \mathrm{m}$ value in a cell. The non-neutralization of the $\mathrm{q} / \mathrm{m}$ in a cell obstructs simultaneous movement of the charged particles at a driving voltage, so that a low driving voltage and reliable lifetime raise issues for an applicable device.

In this study, the effect of the discharging process for electrical neutralization is discussed, and its improved optical property is analyzed with discharging time and hysteresis curve.

\section{EXPERIMENTAL}

Eight sample panels were fabricated using an indium tin oxide(ITO)-coated glass substrate, with barrier ribs formed of $55 \mu \mathrm{m}$ height and $10 \mu \mathrm{m}$ width on the upper and lower substrate, respectively. The upper and lower substrates were designed with the same panel condition, so a cell gap of $110 \mu \mathrm{m}$ was obtained. The negatively charged particles consisted of $\mathrm{TiO}_{2}$ (for white color), negative charge control agents (CCAs), and a polymer; whereas the positively charged particles consisted of carbon black (black color), positive CCAs, and a polymer. The average diameter of the two types of particles was commonly $10 \mu \mathrm{m}$, and their $\mathrm{q} / \mathrm{m}$ value was $-4.5 \mu \mathrm{C} / \mathrm{g}$ and $+4.5 \mu \mathrm{C} / \mathrm{g}$, respectively. 
(a)

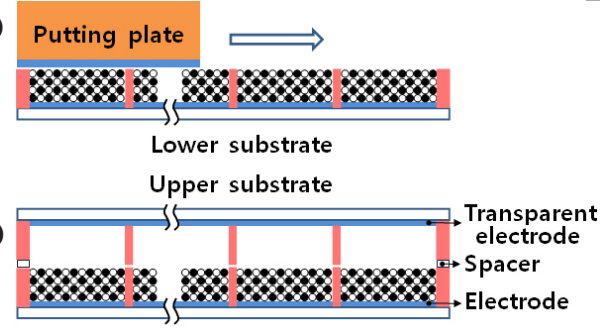

(c)

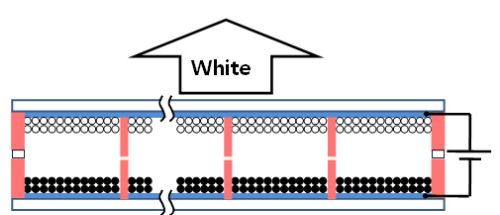

(d)

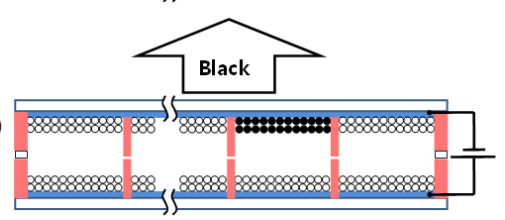

Fig. 1. Fabrication process and realized image of the reflective display (a) simple particle-loading method, (b) packaged panel, (c) white, and (d) black image, by bias electric field.

The electrically opposite particles mixed by an agitator were loaded by a simple particle-loading method, as shown in Fig. 1 (a), which method is reported to obtain a superior performance compared with that of any other methods $[5,6]$. The mixed particles in this particle-loading method were inserted into the cells by mechanical force of the putting plate only, without any electric field [7]. After the particle-loading process, the upper and lower substrates were aligned and packaged, as shown in Fig. 1(b). The quantity of the loaded particles was determined by the height of the barrier ribs on the lower substrate. In this study, the filling ratio of the loaded particles to the total cell volume was about $45 \sim 50 \%$ and this ratio could be controlled by the height of the barrier ribs on the upper substrate and the inserted spacer. This mixed state of the two types of particles is never realized in the normal driving condition, and is a desirable opportunity for particle discharging, for electrical neutralization in a cell. The discharging process for these mixed particles proceeded at a high humidity of $80 \%$ and a high temperature of $30^{\circ} \mathrm{C}$. According to the bias voltage, black and white images were achieved, as shown in Figs. 1(c) and (d).

The above eight panels were fabricated with the same cell condition, and were distinguished only by the different discharging times, of $0,1,2,4,8,16,32$, and 40 days. Reflectivity was measured after these discharging times. To get certain evidence of the improved optical property by discharging process for electrical neutralization of the particles in a cell, a hysteresis curve of the bias voltage for the neutralized panel for a discharging duration of 40 days was compared with that of the non-neutralized panel.

\section{RESULTS AND DISCUSSION}

In the fabrication process of the charged particles, all the particles are designed to get a similar $\mathrm{q} / \mathrm{m}$ value, but this value can be changed during a conveyance, keeping, and particle-loading process. In particular, it is believed that the particle-loading process induces most of the distorted and abnormal electrical charging phenomenon, because of friction with the ribs and particles. It is reported that the charged particles that deviate from (a)

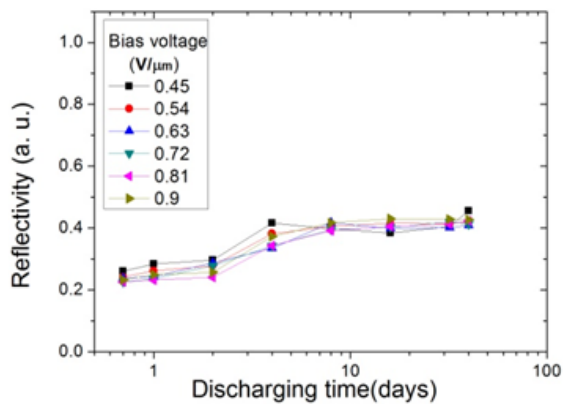

(b)

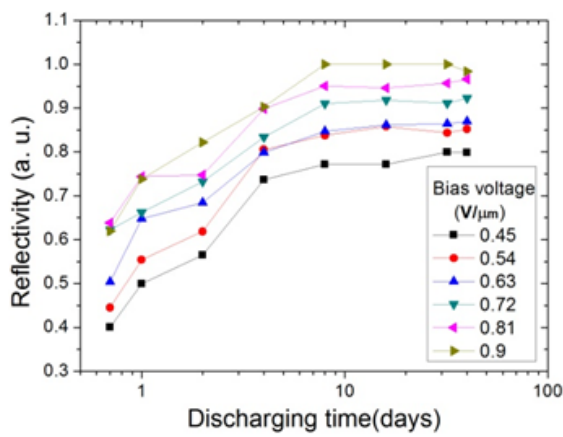

Fig. 2. Reflectivity of the (a) black and (b) white images, according to the discharging times.

the appropriate $\mathrm{q} / \mathrm{m}$ value induce irregular movement, which blocks up simultaneous image transition, resulting in short life time and high driving voltage, with undesirable optical property $[8,9]$. So a recovery process to the original $\mathrm{q} / \mathrm{m}$ value designed at the fabrication process of particles is additionally needed, between processes of panel fabrication. The most simple and certain method for recovery to their intrinsic $\mathrm{q} / \mathrm{m}$ value is an electrical discharging in a mixed state, after the particle-loading process shown in Fig. 1(b). This mixed state is never realized in a normal driving condition. To ascertain the discharging effect, the eight panels, including charged particles of this mixed state, are kept on a thermo-humidistat at a high temperature and humidity. These samples are classified into discharging times, as shown in above section, and for experimental reliability, are not driven before the defined discharging time elapse.

Figure 2 shows reflectivity according to the discharging time. All the mixing ratios of the white to black particles are 1:1, and the reflectivity by a bias electric field is measured immediately after the discharging process. In this figure, the reflectivity of the furthermost left side is that for a non-discharged panel (0 day). Figure 2(a) shows the reflectivity of the black image, and Fig. 2(b) shows that of the white image, depending on discharging time and bias electric field. The reflectivity is measured by the moved particles on the electrode of an upper substrate, so that it represents a quantity of moved particles. As reported in a previous study, the moved particles in the reflective e-paper, QR-LPD, are proportional to the amplitude of the bias electric field [10]. In Fig. 2(a), the variance of the moved black particles depending on a bias electric field is very small, but a slight decrease of the moved black particles depending on the increased discharging time, shows an increase of the reflectivity, which is not intended. The reason is guessed that the negatively charged white particles simultaneously move with a large kinetic energy. However, it is ascertained that the increase depending on the increased discharging time is not large, and is almost saturated after 10 days. In the case of the reflectivity of the white image shown in Fig. 2(b), the reflectivity greatly increases according to the increased discharging time, and is saturated after 10 days, which is reason- 


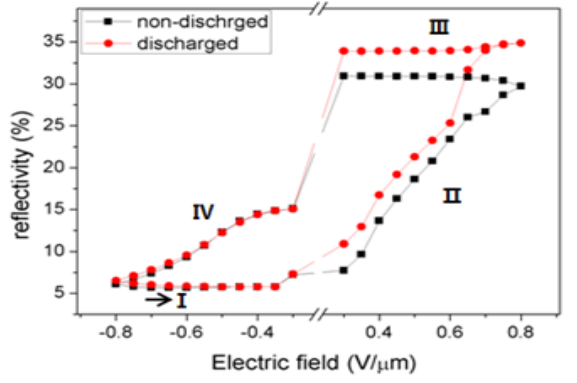

Fig. 3. Hysteresis curve of the non-discharged and discharged particles.

ably understood regarding the result of that of the black image. In consequence, it is believed that an electrical neutralization in a cell by a discharging process at the mixed state of the negatively and positively charged particles has a desirable influence on the optical property.

For an evidence-based approach that the above optical result is induced by stable movement of the particles, and contributes to an improvement of the optical property, two hysteresis curves are obtained, as shown in Fig. 3, for a comparison between two panels having discharged (for 40 days) and non-discharged particles. These hysteresis experiments proceeded by impressing a bias electric field in a counterclockwise direction, and the result for the opposite direction is almost similar. In this figure, the scope of the bias electric field is defined with a saturated reflectivity point, and the unchanged middle region $(-0.25 \sim+0.25$ $\mathrm{V} / \mu \mathrm{m})$ is abbreviated. In region I of the first bias electric field in Fig. 3 , the reflectivity by the discharged black particles is slightly larger than that by the non-discharged particles, as considered in Fig. 2(a). But the reflectivity of the white image by the discharged particles at region III in Fig. 3 greatly increases, as considered in Fig. 2(b). In region II, showing a transition from a black image to a white image, the slope and the absolute value of the reflectivity are larger than that of region IV, showing a reverse image transition. From these results of Figs. 2 and 3, it is believed that the white particles move with a larger kinetic energy than the black particles. To improve the optical property, or to get a similar slope at a color transition, it is believed that an electrical balance of the $\mathrm{q} / \mathrm{m}$ value of the black and white particles is important. The optical property is improved by electrical discharging, and the reason is believed to be an electrical neutralization or stabilization of the particles in a cell. These electrically stable particles will contribute to lowering driving voltage by simultaneous particle movement, and offer a reliable lifetime.

It is desirable that the time-consuming discharging process is replaced by a simple process, for example, by an aging process with high temperature or electrical pulses.

\section{CONCLUSIONS}

In a reflective electronic display using electrically positive and negative particles, the unstable particle movement by non- neutralized $\mathrm{q} / \mathrm{m}$ value induces an undesirable optical property. The non-neutralization of the $\mathrm{q} / \mathrm{m}$ in a cell obstructs simultaneous movement of the charged particles at a driving voltage, so a low driving voltage and reliable lifetime raise an issue for an applicable device. In this study, the effect of a discharging process establishing an electrical neutralization was discussed, and its improved optical property was analyzed with discharging time and hysteresis curve. For this study, eight reflective panels were fabricated with different discharging times, and thrown into the discharging process at high humidity and temperature with a mixed state of negatively and positively particles, and their optical properties were measured. The number of moved black particles according to the increased discharging time decreased slightly, however, the number of the moved white particles greatly increased. As a consequence, it is believed that electrical neutralization in a cell, by a discharging process at a mixed state of negatively and positively charged particles, has a desirable influence on the optical property. The hysteresis curve comparison of two panels with discharged and non-discharged particles, showed an improved optical property by electrical neutralization. These electrically stable particles will contribute to lowering driving voltage with simultaneous particle movement and a reliable lifetime. It is desirable that the time-consuming discharging process be replaced by a simple process, for example, an aging process with high temperature or electrical pulses.

\section{ACKNOWLEDGMENT}

The author would like to thank Chungwoon University for supporting this research through the Research Year Program (2012).

\section{REFERENCES}

[1] D. K. Yang, J. W. Doane, Z. Yaniv and J. Glasser, Appl. Phys. Lett. Vol. 64(15), 1905 (1994) [DOI: http://dx.doi.org/10.1364/ OME.2.001121].

[2] J. S. Kim and Y. C. Kim, J. KIEEME, 24(8), 669 (2011) [DOI: http://dx.doi.org/10.4313/JKEM.2011.24.8.669]

[3] Y. C. Kim, J. TEEM, 13(4), 212 (2012) [DOI: http://dx.doi. org/10.4313/TEEM.2012.13.4.212].

[4] J. S. Kim and Y. C. Kim, J. KIEEME, 25(1), 48 (2012) [DOI: http:// dx.doi.org/10.4313/JKEM.2012.25.1.48]

[5] Y. C. Kim, J. KIEEME, 22(2), 169 (2009) [DOI: http://dx.doi. org/10.4313/JKEM.2009.22.2.169]

[6] C. W. Kim and Y. C. Kim, J. KIEEME, 23(9), 691 (2010) [DOI: http://dx.doi.org/10.4313/JKEM.2010,23.9.691].

[7] D. J. Lee, Y. M. Oh, S. W. Park, B. E. Park and Y. C. Kim, J. DT, 8(6), 361 (2012) [DOI: http://dx.doi.org/10.1109/JDT.2012.2190135].

[8] Y. C. Kim, J. TEEM, 13(5), 233 (2012) [DOI: http://dx.doi. org/10.4313/TEEM.2012.13.5.233].

[9] Y. C. Kim, J. KIEEME, 24(11), 915 (2011) [DOI: http://dx.doi.org/ 10.4313/JKEM.2011.24.11.915].

[10] D. J. Lee and Y. C. Kim, J. KIEEME, 25(2), 129 (2012) [DOI: http://dx.doi.org/10.4313/JKEM.2012.25.2.129]. 